\title{
Extracorporeal Cardiopulmonary Resuscitation (ECPR) Survival: A Quaternary Center Analysis
}

\author{
Lucas Marinacci $^{1}$, Nino Mihatov ${ }^{2}$, David D’Alessandro ${ }^{1}$, Mauricio Villavicencio ${ }^{1}$, Nathali \\ Roy $^{3}$, Yuval Raz ${ }^{1}$, and Sunu Thomas ${ }^{1}$ \\ ${ }^{1}$ Massachusetts General Hospital \\ ${ }^{2}$ New York-Presbyterian/Columbia University Medical Center \\ ${ }^{3}$ Boston Children's Hospital
}

February 11, 2021

\begin{abstract}
Background: Extracorporeal cardiopulmonary resuscitation (ECPR) has emerged as a rescue strategy for non-responders to conventional CPR (CCPR) in cardiac arrest. Definitive guidelines for ECPR deployment do not exist. Prior studies suggest that arrest rhythm and cardiac origin of arrest may be variables used to assess candidacy for ECPR. Aim: To describe a single center experience with ECPR and to assess associations between survival and physician-adjudicated origin of arrest and arrest rhythm. Methods: A retrospective review of all patients who underwent ECPR at a quaternary care center over a 7-year period was performed. Demographic and clinical characteristics were extracted from the medical record and used to adjudicate origin of cardiac arrest, etiology, rhythm, survival, and outcomes. Univariate analysis was performed to determine association of patient and arrest characteristics with survival. Results: Between 2010 and 2017, 47 cardiac arrest patients were initiated on extracorporeal membrane oxygenation (ECMO) at the time of active CPR. ECPR patient survival to hospital discharge was $25.5 \%(\mathrm{n}=12)$. Twenty-six patients died on ECMO $(55.3 \%)$ while 9 patients $(19.1 \%)$ survived decannulation but died prior to discharge. Neither physician-adjudicated arrest rhythm nor underlying origin were significantly associated with survival to discharge, either alone or in combination. Younger age was significantly associated with survival. Nearly all survivors experienced myocardial recovery and left the hospital with a good neurological status. Conclusions: Arrest rhythm and etiology may be insufficient predictors of survival in ECPR utilization. Further multi-institutional studies are needed to determine evidenced based criteria for ECPR deployment.
\end{abstract}

Extracorporeal Cardiopulmonary Resuscitation (ECPR) Survival: A Quaternary Center Analysis

Lucas X. Marinacci ${ }^{1 *}$, Nino Mihatov $\mathrm{MD}^{2 *}$, David A D'Alessandro $\mathrm{MD}^{3}$, Mauricio Villavicencio $\mathrm{MD}^{3}$, Nathalie Roy $\mathrm{MD}^{4}$, Yuval Raz ${ }^{5}$, Sunu S Thomas MD, MSc${ }^{1,6}$

1. Department of Medicine, Massachusetts General Hospital, Boston, MA

2. Division of Cardiology, New Yorl-Presbyterian/Columbia University Medical Center

3. Division of Cardiac Surgery, Massachusetts General Hospital, Boston, MA

4. Department of Cardiac Surgery, Boston Children's Hospital, Boston, MA

5. Division of Pulmonary and Critical Care, Massachusetts General Hospital, Boston, MA

6. Cardiology Division, Massachusetts General Hospital, Boston, MA

Running Head: ECPR Survival by Arrest Origin and Rhythm

\section{Address for Correspondence:}

Sunu S. Thomas, MD, MSc 
Cardiology Division, Massachusetts General Hospital

55 Fruit Street; Bigelow 800

Boston, MA 02114

sunu.thomas@mgh.harvard.edu

Phone: 617-643-7249

Fax: $617-724-4105$

*Drs. Marinacci and Mihatov contributed equally to this work.

Disclosures: NM receives funding from the National Heart, Lung and Blood Institute (grant T32HL007208).

Funding: None

Data Sharing : The data that support the findings of this study are available on request from the corresponding author. The data are not publicly available due to privacy or ethical restrictions.

\section{Abstract}

Background : Extracorporeal cardiopulmonary resuscitation (ECPR) has emerged as a rescue strategy for non-responders to conventional CPR (CCPR) in cardiac arrest. Definitive guidelines for ECPR deployment do not exist. Prior studies suggest that arrest rhythm and cardiac origin of arrest may be variables used to assess candidacy for ECPR.

Aim : To describe a single center experience with ECPR and to assess associations between survival and physician-adjudicated origin of arrest and arrest rhythm.

Methods : A retrospective review of all patients who underwent ECPR at a quaternary care center over a 7-year period was performed. Demographic and clinical characteristics were extracted from the medical record and used to adjudicate origin of cardiac arrest, etiology, rhythm, survival, and outcomes. Univariate analysis was performed to determine association of patient and arrest characteristics with survival.

Results : Between 2010 and 2017, 47 cardiac arrest patients were initiated on extracorporeal membrane oxygenation (ECMO) at the time of active CPR. ECPR patient survival to hospital discharge was $25.5 \%$ $(\mathrm{n}=12)$. Twenty-six patients died on ECMO $(55.3 \%)$ while 9 patients $(19.1 \%)$ survived decannulation but died prior to discharge. Neither physician-adjudicated arrest rhythm nor underlying origin were significantly associated with survival to discharge, either alone or in combination. Younger age was significantly associated with survival. Nearly all survivors experienced myocardial recovery and left the hospital with a good neurological status.

Conclusions : Arrest rhythm and etiology may be insufficient predictors of survival in ECPR utilization. Further multi-institutional studies are needed to determine evidenced based criteria for ECPR deployment.

Introduction :

Sudden cardiac arrest in adults has low survival rates despite the widespread implementation of cardiopulmonary resuscitation $(\mathrm{CPR})^{1}$. Hospital cardiac arrest survival to discharge can be less than $20 \%^{2-4}$. Moreover, the odds of survival decline with increasing duration of $\mathrm{CPR}^{5,6}$.

Extracorporeal membrane oxygenation (ECMO) during CPR, referred to as ECPR, can provide complete cardiopulmonary support and has emerged as a strategy to provide temporary perfusion and oxygenation for patients in cardiac arrest. The data in support of ECPR over conventional CPR (CCPR) remain mixed and are limited by the heterogeneity of a small number of studies.

Propensity matched observational studies comparing ECPR with CCPR suggest improved in-hospital and 1-year survival, as well as improved neurological outcomes with $\mathrm{ECPR}^{7-10}$. A large meta-analysis found that for adult patients with in-hospital cardiac arrest thought to be of cardiac origin, ECPR was associated with 
significantly improved survival and neurological outcomes compared to CCPR ${ }^{11}$. Others, however, have found no clear benefit in survival to hospital discharge with ECPR ${ }^{12}$.

There are no definitive initiation criteria for ECMO deployment during in-hospital CPR. Among patients who undergo ECPR, approximately $20-40 \%$ survive to discharge, with younger age being a potential predictor for favorable neurological outcome ${ }^{13-20}$. Factors that have been reported to correlate with survival in ECPR include younger age ${ }^{17}$, shorter CPR duration ${ }^{16,21}$, normal pre-cannulation renal function ${ }^{22}$, a simplified acute physiology score II of less than $80^{23}$, a serum lactate less than $4.6 \mathrm{mmol}$ per liter ${ }^{24}$, or a diagnosis of acute myocarditis ${ }^{19}$.

The prognostic significance of the arrest rhythm, arrest etiology, and the delineation of cardiac versus non-cardiac origin of cardiac arrest in ECPR remains controversial. A meta-analysis of 856 patients who underwent ECPR for in hospital cardiac arrest demonstrated that an initial shockable rhythm was associated with survival. However, no significant association was found between survival and cardiac versus non-cardiac origin of arrest ${ }^{25}$. In contrast, a comparison of ECPR and CCPR found a higher rate of intensive care unit survival and long term favorable neurological outcome in ECPR patients with a non-cardiac origin of arrest ${ }^{10}$. Other studies report survival rates of $20 \%$ in patients with non-shockable rhythm at time of ECPR, suggesting a shockable rhythm should not be a solitary prerequisite for ECPR, however information on cardiac arrest origin or etiology was not included ${ }^{26}$.

In light of the expanding utilization of ECPR and the lack of definitive criteria for optimal patient selection, we performed a single center retrospective observational study in hospitalized patients with a cardiac arrest who received ECPR. With this analysis, we sought to determine the association between arrest survival and the predominant arrest rhythm and underlying etiology in each case by physician-adjudication chart review, and defined each cardiac arrest as cardiac or non-cardiac in origin based on the etiology.

\section{Methods and Materials:}

A retrospective cohort review was performed on all entries in the Extracorporeal Life Support Organization (ELSO) registry from November 1, 2010 to July 31, 2017 at the Massachusetts General Hospital (MGH), Boston, Massachusetts (USA). Patients who were undergoing CPR in hospital at the time of ECMO initiation as documented in the procedural report were included. Patients who underwent ECMO cannulation but for whom immediate durable flow through the ECMO circuit could not be initiated were excluded. Baseline characteristics were extracted from the medical record. The study was approved by the Partners Institutional Review Board.

This study's primary outcome was survival to hospital discharge. Etiology of cardiac arrest was adjudicated by two reviewers (NM \& LXM) and categorized by cardiac origin (defined as an etiology of acute coronary syndrome, primary arrhythmia in the absence of overlapping etiology, acute heart failure, and tamponade) or non-cardiac origin (defined as pulmonary embolism, drug toxicity, sepsis, perioperative shock, or air embolism). When multiple potential etiologies were present, the primary cause as adjudicated by the care team was selected. Cardiac arrest rhythm was ascertained from the procedural report and categorized as either pulseless ventricular tachycardia/ventricular fibrillation (VT/VF) or pulseless electrical activity (PEA). CCPR time, defined as duration of chest compressions immediately prior to ECMO initiation, was reported when available.

At our institution, absolute contraindications to ECMO support include acute intracranial hemorrhage or massive stroke, contraindication to anticoagulation or refusal to receive blood products, active bleeding, and severe aortic insufficiency. Relative contraindications included age greater than 70 years, active cancer, suicide attempt, chronic hemodialysis, lack of social support or healthcare proxy, CCPR greater than 45 minutes, aortic dissection, end stage liver disease, and body-mass index greater than $45 \mathrm{~kg} / \mathrm{m}^{2}$.

ECMO-related complications were assessed. Major bleeding was defined as Bleeding Academic Research Consortium category $3-527$. Additional outcomes assessed included de novo renal replacement therapy, cerebrovascular events, limb ischemia, and cerebral performance category (CPC). CPC scores 1 through 5 
correlated to good cerebral performance (able to work, minor deficits), moderate cerebral disability (persistent deficits but mostly independent), severe cerebral disability (dependent on others), coma or vegetative state, and brain death, respectively ${ }^{28}$.

Descriptive statistics were used to quantify baseline patient characteristics, clinical features, ECMO utilization, and survival rates. Categorical variables were reported as counts and percentages and compared using the Fisher's exact test. Continuous variables were reported as medians with interquartile range (IQR) and compared with the Wilcoxon two-sample test. Statistical significance was assessed at a nominal $\alpha$ level of 0.05. All reported P values were 2-sided. Analysis was performed in R 4.0.0 (R Core Team, 2020).

\section{Results:}

Between November 2010 and September 2017, 47 cardiac arrest patients were initiated on ECMO at the time of active CPR for cardiac arrest. Baseline characteristics for survivors and non-survivors are described in Table 1. ECPR patients were more commonly male $(\mathrm{n}=33 ; 70.2 \%)$ with a median age of 53 years. The majority of patients had underlying cardiovascular disease, including 20 patients (42.6\%) with known cardiomyopathy and $27(57.4 \%)$ with coronary artery disease.

Forty-five patients $(95.7 \%)$ experienced an in-hospital cardiac arrest while two patients experienced an outof-hospital arrest but were cannulated in the hospital while receiving CPR (4.3\%).

Arrest rhythms were either PEA $(\mathrm{n}=25 ; 53.2 \%)$ or pulseless VT/VF $(\mathrm{n}=22 ; 46.8 \%)$. Arrest etiologies included acute coronary syndromes $(n=17 ; 36.2 \%)$, acute heart failure syndromes $(n=10 ; 21.3 \%)$, pulmonary embolism $(n=7 ; 14.9 \%)$, primary arrhythmia $(n=5 ; 10.6 \%)$, drug toxicity or overdose $(n=2 ; 4.3 \%)$, and other causes $(\mathrm{n}=6 ; 13 \%)$, including tamponade $(\mathrm{n}=2)$, air embolism $(\mathrm{n}=1)$, perioperative hemorrhagic shock $(\mathrm{n}=1)$, and sepsis/endocarditis $(\mathrm{n}=2)$. In total, 4 patients $(8.5 \%)$ experienced arrest post-cardiotomy (from tamponade, PE, VT storm, and VF), with $50 \%$ of these patients dying while on ECMO and the remaining surviving to discharge. CCPR time was obtained for 40 of the 47 (85.1\%) ECPR patients. Duration of CCPR prior to initiation of ECMO was no different between the 32 non-survivors (40.00 min [IQR 15.00, $54.24]$ ) and the 8 survivors (48.50 min [IQR 27.50, 73.75]), $\mathrm{p}=0.236$ ).

Overall, 34 patients $(72.3 \%)$ were adjudicated to have a primary cardiac origin of their cardiac arrest. There was no significant association found between etiology of arrest, arrest rhythm, or cardiac versus non-cardiac origin of arrest and survival to discharge. Similarly, no combination of arrest origin (cardiac or non-cardiac) and arrest rhythm (PEA or VT/VF) was significantly associated with survival to discharge [Table 2].

ECMO characteristics are described in Table 3. Almost all patients underwent peripheral cannulation. Distal perfusion cannulas were placed if there was clinical evidence of limb ischemia. Following cannulation, 22 (46.8\%) were underwent targeted temperature management, 19 (40.4\%) had a subsequent revascularization procedure, and $6(12.8 \%)$ had a venting ventricular assist device placed. Median time on ECMO support was 3.00 days.

ECMO-related complications included major bleeding $(\mathrm{n}=28 ; 59.6 \%)$, renal replacement therapy $(\mathrm{n}=24$; $51.1 \%)$, cerebrovascular accident $(\mathrm{n}=10 ; 21.3 \%)$, and limb ischemia $(\mathrm{n}=8 ; 17.0 \%)$ [Table 4].

Overall ECPR patient survival to hospital discharge was $25.5 \%(\mathrm{n}=12)$. Twenty-six patients died on ECMO $(55.3 \%)$ while 9 patients $(19.1 \%)$ survived ECMO decannulation but died prior to discharge. The majority of patients who survived to discharge experienced myocardial recovery $(\mathrm{n}=10 ; 83 \%)$; one patient underwent orthotopic heart transplantation (8.3\%), one received a durable ventricular assist device (8.3\%), and 2 required new dialysis at discharge $(16.7 \%)$. Most survivors had a favorable CPC score of 1 at discharge $(\mathrm{n}=9 ; 66.7 \%)$ [Table 5]. Median intensive care unit and hospital length of stay for survivors was 28.5 [IQR $13.50,39.50]$ and 35.50 days [IQR 21.75, 49.50] respectively. Causes of death in non-survivors are listed in Table 6 .

In univariate analysis, characteristics associated with survival to discharge include younger age (median 44.00 [IQR 45.00,64.00] in survivors versus 60.00 in non-survivors [IQR 46.00, 6.65]; p 0.034) and arrest location 
in the emergency department (33.3\% of survivors versus $2.9 \%$ of non-survivors; $p=0.012$ ). Arrest etiology of pulmonary embolism approached but did not cross the pre-established threshold for significance $(33.3 \%$ of survivors versus $8.6 \%$ of non-survivors, $\mathrm{p}=0.060$ ).

\section{Discussion:}

In this single-center study, we demonstrate that physician adjudicated arrest rhythm (VT/VF versus PEA) or underlying origin of the cardiac arrest (cardiac versus non-cardiac) were not significantly associated with survival to hospital discharge following ECPR, either alone or in combination. Of the multiple baseline, peri-arrest, and on-circuit characteristics assessed, only younger age was significantly associated with survival following ECPR. Of those cardiac arrest etiologies evaluated, only pulmonary embolism approached statistical significance for the primary outcome of survival to hospital discharge. We found that ECPR therapy was associated with a high complication burden, with the majority of patients experiencing major bleeding or requiring de novo renal replacement therapy. Nearly all ECPR survivors to discharge experienced myocardial recovery, in addition to a CPC score consistent with a good functional neurological status. Of the patients who did not survive, the most common cause of death was the decision to pursue comfort measures in the setting of multiorgan failure.

The initiation of ECMO at the time of in-hospital cardiac arrest with active CPR remains an evolving treatment strategy without a robust evidence base to guide appropriate patient selection. Our study had the advantage of a detailed physician review of cardiac arrest origin, etiology, and rhythm and highlights the heterogeneity in adjudicated causes of cardiac arrest preceding ECPR initiation. This study corroborates the existing literature that underscores the salient challenge in predicting survival following ECPR attributed to the complexity and heterogeneity of the cardiac arrest event.

As rescue ECPR utilization expands, patient selection remains a critical determinant in ensuring the appropriate implementation of a resource-intense therapy to ensure clinical benefit. Our results suggest that younger age and a cardiac arrest etiology attributed to pulmonary embolism may be associated with survival in ECPR. In pulmonary embolism cases, ECMO affords both critical cardiopulmonary support in addition to obligate anticoagulation that may relieve a potentially reversible pulmonary vascular obstruction leading to an arrest. Younger age may speak to the physiologic reserve required to tolerate not just the arrest event itself and attendant ischemic risk, but also the subsequent ECMO therapy and its potential high complication burden. Nevertheless, the overall high functional status of those who survive to discharge, despite the high frequency of significant underlying conditions as described in Table 1, may justify the use of this therapy even in patients with multiple pre-existing medical problems.

There are inherent limitations to this study attributed to a retrospective analysis, sample size, and a dependence on the quality of clinical record keeping at the time of each ECPR event. Conventional ACLS resuscitation algorithms are delineated by a cardiac arrest's rhythm and etiology. However, our analysis suggests that as an initial trigger point, ECPR outcomes are not dependent on the arrest rhythm or etiology. It may be that at the time of ECMO initiation, the consequent severity of the arrest's cardiopulmonary failure may render a survivable ECPR outcome futile. To this end, metrics that could provide additional insight into the relationship between the quality of the initial arrest resuscitation efforts and ECPR survival, including end-tidal $\mathrm{CO}_{2}$ prior to ECMO initiation and the frequency and duration of ROSC could not be reliably obtained. However, our study did find that in the 40 patients for whom low-flow time was recorded, the duration of mechanical CPR was not associated with ECPR survival outcomes.

Moreover, the current findings do not account for potential improvements in ECPR initiation and postECPR care over the study period. The current registry does not include cardiac arrest patients for whom ECMO support was considered but ultimately not provided. While emblematic of the challenges with ECPR studies, our findings should be viewed as primarily hypothesis generating and complimentary to the existing evidence base with similar limitations.

It may be that arrest origin, etiology, and rhythm gain prognostic significance when considered alongside resuscitation metrics. For example, there is evidence that taking into account initial rhythm along with 
low-flow time could help predict neurological outcomes ${ }^{1,31}$. Ultimately, the potential for ECMO as a rescue strategy may be limited at its onset by the extent of ischemic damage suffered prior to it its initiation; if adequately characterized, this could define the upper limit to therapeutic benefit of ECPR and thereby inform point of care decision making. Future prospective studies of ECPR should focus on combinations of variables which might capture the depth and severity of ischemic insults sustained in the period after arrest but before ECMO deployment, especially those which are readily attainable and have the potential to be easily incorporated into future advanced cardiovascular life support protocols.

\section{Conclusion:}

In our study, ECPR survival to hospital discharge was not associated with the origin or presenting rhythm of a cardiac arrest. Younger patients and arrested from a pulmonary embolism may represent a population who benefits from ECPR that warrants future study. Prospective studies that further evaluate pre-arrest patients characteristics and standardized ECPR protocols are warranted.

\section{References}

1. Cooper, S., Janghorbani, M. \& Cooper, G. A decade of in-hospital resuscitation: outcomes and prediction of survival? Resuscitation 68, 231-237 (2006).

2. Ebell, M. H., Becker, L. A., Barry, H. C. \& Hagen, M. Survival after in-hospital cardiopulmonary resuscitation. A meta-analysis. J. Gen. Intern. Med. 13, 805-816 (1998).

3. Peberdy, M. A. et al. Cardiopulmonary resuscitation of adults in the hospital: A report of 14720 cardiac arrests from the National Registry of Cardiopulmonary Resuscitation. Resuscitation vol. 58 297-308 (2003).

4. Goldberger, Z. D. et al. Duration of resuscitation efforts and survival after in-hospital cardiac arrest: an observational study. Lancet 380, 1473-1481 (2012).

5. Schultz, S. C., Cullinane, D. C., Pasquale, M. D., Magnant, C. \& Evans, S. R. T. Predicting in-hospital mortality during cardiopulmonary resuscitation.Resuscitation vol. 33 13-17 (1996).

6. Bartos, J. A. et al.Improved Survival with Extracorporeal Cardiopulmonary Resuscitation Despite Progressive Metabolic Derangement Associated with Prolonged Resuscitation. Circulation CIRCULATIONAHA.119.042173 (2020).

7. Chen, Y.-S. et al.Cardiopulmonary resuscitation with assisted extracorporeal life-support versus conventional cardiopulmonary resuscitation in adults with in-hospital cardiac arrest: an observational study and propensity analysis. The Lancet vol. 372 554-561 (2008).

8. Shin, T. G. et al.Extracorporeal cardiopulmonary resuscitation in patients with inhospital cardiac arrest: A comparison with conventional cardiopulmonary resuscitation. Crit. Care Med. 39, 1-7 (2011).

9. Shin, T. G. et al.Two-year survival and neurological outcome of in-hospital cardiac arrest patients rescued by extracorporeal cardiopulmonary resuscitation.Int. J. Cardiol. 168, 3424-3430 (2013).

10. Patricio, D. et al.Comparison of extracorporeal and conventional cardiopulmonary resuscitation: a retrospective propensity score matched study.Crit. Care 23, 27 (2019).

11. Ahn, C. et al.Efficacy of extracorporeal cardiopulmonary resuscitation compared to conventional cardiopulmonary resuscitation for adult cardiac arrest patients: a systematic review and meta- analysis. Nature Publishing Group (2016) doi:10.1038/srep34208.

12. Kim, S. J., Kim, H. J., Lee, H. Y., Ahn, H. S. \& Lee, S. W. Comparing extracorporeal cardiopulmonary resuscitation with conventional cardiopulmonary resuscitation: A meta-analysis. Resuscitation vol. 103 106-116 (2016).

13. Axtell, A. L. et al. Predictors of Neurologic Recovery in Patients Who Undergo Extracorporeal Membrane Oxygenation for Refractory Cardiac Arrest.J. Cardiothorac. Vasc. Anesth. 34, 356-362 (2020). 
14. Lunz, D. et al.Extracorporeal membrane oxygenation for refractory cardiac arrest: a retrospective multicenter study. Intensive Care Med. (2020) doi:10.1007/s00134-020-05926-6.

15. Ryu, J.-A. et al.Neurologic Outcomes in Patients Who Undergo Extracorporeal Cardiopulmonary Resuscitation. Ann. Thorac. Surg. 108, 749-755 (2019).

16. Chen, Y. S. et al.Analysis and results of prolonged resuscitation in cardiac arrest patients rescued by extracorporeal membrane oxygenation. J. Am. Coll. Cardiol. 41, 197-203 (2003).

17. Cardarelli, M. G., Young, A. J. \& Griffith, B. Use of Extracorporeal Membrane Oxygenation for Adults in Cardiac Arrest (E-CPR): A Meta-Analysis of Observational Studies. ASAIO J. 55, 581-586 (2009).

18. Peigh, G., Cavarocchi, N. \& Hirose, H. Saving life and brain with extracorporeal cardiopulmonary resuscitation: A single-center analysis of in-hospital cardiac arrests.J. Thorac. Cardiovasc. Surg. 150, 1344-1349 (2015).

19. Thiagarajan, R. R.et al. Extracorporeal membrane oxygenation to support cardiopulmonary resuscitation in adults. Ann. Thorac. Surg.87, 778-785 (2009).

20. Richardson, A. S. C.et al. ECMO Cardio-Pulmonary Resuscitation (ECPR), trends in survival from an international multicentre cohort study over 12-years. Resuscitation 112, 34-40 (2017).

21. Chen, Y.-S. et al.Extracorporeal membrane oxygenation support can extend the duration of cardiopulmonary resuscitation. Crit. Care Med. 36, 2529-2535 (2008).

22. Mendiratta, P. et al. Cardiopulmonary Resuscitation Requiring Extracorporeal Membrane Oxygenation in the Elderly. ASAIO J. 59, 211-215 (2013).

23. Kim, K. I. et al.The pre-ECMO simplified acute physiology score II as a predictor for mortality in patients with initiation ECMO support at the emergency department for acute circulatory and/or respiratory failure: a retrospective study. Scand. J. Trauma Resusc. Emerg. Med.23, 59 (2015).

24. Jung, C. et al.Outcome predictors in cardiopulmonary resuscitation facilitated by extracorporeal membrane oxygenation. Clin. Res. Cardiol.105, 196-205 (2016).

25. D'Arrigo, S. et al.Predictors of favourable outcome after in-hospital cardiac arrest treated with extracorporeal cardiopulmonary resuscitation: A systematic review and meta-analysis. Resuscitation 121, 62-70 (2017).

26. Pabst, D. \& Brehm, C. E. Is pulseless electrical activity a reason to refuse cardiopulmonary resuscitation with ECMO support? Am. J. Emerg. Med. 36, 637-640 (2018).

27. Mehran Roxana et al. Standardized Bleeding Definitions for Cardiovascular Clinical Trials. Circulation 123, 2736-2747 (2011).

28. A randomized clinical study of cardiopulmonary - cerebral resuscitation: Design, methods, and patient characteristics. Am. J. Emerg. Med. 4, 72-86 (1986).

29. Meaney, P. A. et al. Rhythms and outcomes of adult in-hospital cardiac arrest*. Read Online: Critical Care Medicine | Society of Critical Care Medicine 38, 101 (2010).

30. Fagnoul, D. et al.Extracorporeal life support associated with hypothermia and normoxemia in refractory cardiac arrest. Resuscitation 84, 1519-1524 (2013).

31. Ko, R. E. et al.The differential neurologic prognosis of low-flow time according to the initial rhythm in patients who undergo extracorporeal cardiopulmonary resuscitation. Resuscitation 148, 121-127 (2020).

\section{Hosted file}

Table 1.pdf available at https://authorea.com/users/336216/articles/508474-extracorporealcardiopulmonary-resuscitation-ecpr-survival-a-quaternary-center-analysis 


\section{Hosted file}

Table 2.pdf available at https://authorea.com/users/336216/articles/508474-extracorporealcardiopulmonary-resuscitation-ecpr-survival-a-quaternary-center-analysis

\section{Hosted file}

Table 3.pdf available at https://authorea.com/users/336216/articles/508474-extracorporealcardiopulmonary-resuscitation-ecpr-survival-a-quaternary-center-analysis

\section{Hosted file}

Table 4.pdf available at https://authorea.com/users/336216/articles/508474-extracorporealcardiopulmonary-resuscitation-ecpr-survival-a-quaternary-center-analysis

\section{Hosted file}

Table 5.pdf available at https://authorea.com/users/336216/articles/508474-extracorporealcardiopulmonary-resuscitation-ecpr-survival-a-quaternary-center-analysis

\section{Hosted file}

Table 6.pdf available at https://authorea.com/users/336216/articles/508474-extracorporealcardiopulmonary-resuscitation-ecpr-survival-a-quaternary-center-analysis 